\title{
siRNA enrichment in Argonaute 2-depleted Blattella germanica
}

\author{
José Carlos Montañés ${ }^{\mathrm{a}, 1}$, Carlos Rojano ${ }^{\mathrm{a}}$, Guillem Ylla ${ }^{\mathrm{b}, *}$, Maria Dolors Piulachs ${ }^{\mathrm{a}, *}$, \\ José Luis Maestro ${ }^{\text {a,* }}$ \\ ${ }^{a}$ Institute of Evolutionary Biology (CSIC-Universitat Pompeu Fabra), Passeig Marítim de la Barceloneta 37-49, 08003 Barcelona, Spain \\ ${ }^{\mathrm{b}}$ Department of Organismic and Evolutionary Biology, Harvard University, Cambridge, MA, USA
}

\section{A R T I C L E I N F O}

\section{Keywords:}

RNAi

Argonaute

dsRNA

Insect

Blattella germanica

\begin{abstract}
A B S T R A C T
Background: RNA interference (RNAi) is a cellular mechanism used to fight various threats, including transposons, aberrant RNAs, and some types of viruses. This mechanism relies on the detection of dsRNA molecules, which through a pathway involving Dicer-2 (Dcr-2) and Argonaute 2 (AGO2), produces small interfering RNAs (siRNAs) that bind to the complementary RNAs triggering their degradation.

Methods: Using the cockroach Blattella germanica as a model, we examined AGO2 activity by depleting its mRNA using RNAi and analyzing the phenotypes produced.

Results: Depleting AGO2 expression had no remarkable effect on nymphal development or reproduction. dsRNA treatment triggered an immediate and transitory increase in AGO2 expression, independently of Dcr-2 action. In addition, we analyzed the siRNAs generated after injecting a heterologous dsRNA in control and AGO2-depleted animals. The results revealed that obtained siRNAs mapped non-uniformly along the dsRNA sequence. In AGO2depleted animals, the proportion of 22 nucleotide reads was higher and accumulations of reads appeared in areas less well-represented in the controls. We also detected a preference for cytosine as the first nucleotide in controls that was significantly attenuated in AGO2-depleted individuals.

Conclusions/general significance: The siRNAs produced from a dsRNA mapped heterogeneously along the length of the dsRNA and this arrangement depends on the dsRNA sequence. AGO2 exerts its role as nuclease on the siRNA duplexes independently of its action on the corresponding mRNA. This study sheds light on an extremely useful process for reverse genetics in laboratories, in addition to the design of more effective, specific, and eco-friendly pest-control strategies.
\end{abstract}

\section{Introduction}

RNA interference (RNAi) is a mechanism that cells use to defend themselves against certain types of aggression, both internal and external. The cells detect the presence of double-stranded RNA (dsRNA) from certain types of viruses, transposons, and endogenous dsRNA from hairpin-like RNA secondary structures or bidirectional transcription [1]. A series of enzymatic reactions are activated to degrade the dsRNA and eliminate it.

Researchers can exploit the RNAi mechanism to perform functional genomics studies. To do this, the organism under study is treated with a dsRNA containing the sequence of a given mRNA. The cellular machinery of the treated individual processes the dsRNA using its RNAi pathway and triggers the degradation of the targeted mRNA, leading to a concomitant reduction of the protein it encodes. The resulting phenotypes provide information on the functional role of the knocked down protein [2]. Additionally, RNAi methods using several delivery strategies are being implemented for pest control purposes [3]. These strategies include transgenic crops expressing dsRNA, applying dsRNA to leaves or soil, delivering dsRNA via non-pathogenic insect viruses, and feeding liposome-encapsulated dsRNA [4,5].

The RNAi mechanism starts with the activation of a ribonuclease type III called Dicer (Dcr; Dcr-2 in the case of insects. Dcr-2 expression is activated by the treatment with a dsRNA as short as $50 \mathrm{bp}$, but not by a similar length dsDNA fragment [6]. Dcr-2 cleaves the dsRNA, producing duplexes between 21 and 23 nt with two nucleotides overhanging at each $3^{\prime}$ end. These small double-stranded RNAs are referred to as small interfering RNAs (siRNAs) [1]. The siRNA duplexes are then bound to the dsRNA binding protein R2D2, which, together with Dcr-2, transfers siRNA duplexes into the RNA-induced silencing complex (RISC). There,

\footnotetext{
* Corresponding authors.

E-mail addresses: guillem_ylla@fas.harvard.edu (G. Ylla), mdolors.piulachs@ibe.upf-csic.es (M.D. Piulachs), joseluis.maestro@ibe.upf-csic.es (J.L. Maestro).

1 Present address: Research Unit on Biomedical Informatics, IMIM (Hospital del Mar Medical Research Institute), Barcelona, Spain.
} 
one of the siRNA strands is kept (guide strand), while the other strand (passenger strand) is cut and released [7]. Using the siRNA guide strand as a template, the RISC finds the target RNA through complementary base pairing and cleaves it, leading to its degradation [1].

The cleaving activity of both the passenger strand and complementary RNA is implemented by the core component of the RISC, a nuclease known as Argonaute (AGO). Insects have different AGO proteins. The proteins AGO3 and Piwi are related to the piRNA pathway [8]. On the other hand, AGO1 is used in the micro RNA (miRNA) pathway, whereas AGO2 is used in the siRNA pathway, although may sometimes function in the miRNA pathway, particularly in the absence of AGO1 [9]. The loading of siRNA duplexes into the RISC is of the utmost importance as this determines which of the strands becomes the guide strand, and it is the role of the guide strand to identify the target RNAs by complementarity. Only if a RNA sequence contains a complementary sequence to the guide strand, this can be targeted for degradation by the siRNA.

The action of AGO2 at the cellular level in insects has primarily been studied in Drosophila melanogaster, in which it has been shown that AGO2 binds to small RNAs (approx. $21 \mathrm{nt}$ ) originating from retrotransposons and long stem-loop structures from repetitive sequences in the genome [10]. In addition, AGO2 has been shown to be solely responsible for target RNA cleavage activity guided by siRNAs [11]. The site of cleavage of the target RNA is defined by the $5^{\prime}$ end of the guide strand and located near the center of the guiding sequence $[12,13]$. In the cockroach Blattella germanica, depleting AGO2 reduces the silencing activity of specific dsRNAs [9].

The organism of choice for our experiments is the German cockroach B. germanica a hemimetabolous insect, that undergoes molting to an adult from the last juvenile (nymphal) stage without having to go through a pupal stage. In this species, we have already described the roles of AGO1 and AGO2 in the miRNA pathway [9] and demonstrated its extremely high sensitivity to RNAi $[14,15]$. Here, we analyze the regulation of $A G O 2$ expression and its function at different levels. The imaginal molt, as well as the oviposition, are processes very sensitive to diverse disturbances [16-18]. Therefore, the measurement of the duration of the last nymphal instar and the reproductive cycle and the offspring number will give us an idea of the affectation produced by the RNAi-directed AGO2 depletion. Furthermore, to analyze the contribution of AGO2 to the formation and maintenance of siRNAs produced from dsRNA, we injected a heterologous dsRNA in control and AGO2depleted animals, and analyzed the resultant siRNAs. The heterologous dsRNA has no target mRNA in the cells, allowing us to study the cellular mechanisms of dsRNA processing dissociated from the effect of the siRNAs on the target mRNAs.

\section{Material and methods}

\subsection{Insects sampling}

Specimens of $B$. germanica were obtained from a colony reared on dog food and water at $29 \pm 1{ }^{\circ} \mathrm{C}$ and $60-70 \%$ relative humidity. In the experiments to study the duration of the gonadotrophic cycle and the number of offspring, the control and treated females were coupled with males once they molted into adults. All dissections were carried out on carbon dioxide-anaesthetized insects.

\section{2. dsRNA synthesis and B. germanica treatment}

Systemic RNAi treatments were performed as previously described $[17,19]$. The primers used to generate the dsRNA against $B$. germanica Dcr-2 (dsDcr-2) and AGO2 (dsAGO2) are already described [6,9].

Two different heterologous dsRNAs were used throughout the work. The first of them was designed against the polyhedrin of the Autographa californica nucleopolyhedrovirus (dsPolyh), as previously described [9]. The second one was a dsRNA against a $128 \mathrm{bp}$ fragment of the vector pSTBlue-1 (Novagen) (dspSTBlue) [20]. The sequences of the cDNAs that were used to synthesize dsPolyh, ds $A G O 2$ and dsDcr-1, for which we analyzed the siRNAs, are described in the Supplementary Data 1.

In the experiments designed for identifying the functions of AGO2 in development and reproduction, we treated freshly emerged last (sixth) instar female nymphs (N6D0) or freshly emerged adult females (AdD0) with $2 \mu \mathrm{g}$ of dsAGO2 or with $2 \mu \mathrm{g}$ of dsPolyh as a negative control.

To evaluate the changes in the expression of Dcr-2 and AGO2 that are induced by a dsRNA treatment, regardless of the target sequence, we treated female nymphs on the 4th day of the penultimate instar (N5D4) with $2 \mu \mathrm{g}$ of a heterologous dsRNA (dsPolyh) or with the equivalent volume of water as a negative control. The mRNA levels of AGO2 and Dcr-2 were measured 4, 8 and $16 \mathrm{~h}$ later.

To check if the immediate and transient increase of AGO2 expression observed after dsRNA treatment requires Dcr- 2 activity, we replicated the previous experiment but having a control group and a group with Dcr-2 depleted by a previous dsRNA treatment. Thus, the experimental protocol was as follows: we treated female nymphs freshly emerged to the penultimate instar (N5D0) with $2 \mu$ g of dsDcr-2, for depleting Drc-2, or with $2 \mu \mathrm{g}$ of dspSTBlue as a negative control. Four days later, individuals of both treatments were injected with $2 \mu \mathrm{g}$ of dsPolyh or water, and Dcr-2 and AGO2 mRNA levels were quantified $8 \mathrm{~h}$ later.

\subsection{B. germanica small RNA libraries}

B. germanica female nymphs recently emerged into the penultimate instar (N5D0) were treated with $2 \mu \mathrm{g}$ of dspSTBlue (Control insects) or against AGO2 (dsAGO2). Five days later (N5D5), both Control and dsAGO2 animals were treated with $2 \mu \mathrm{g}$ of a second heterologous dsRNA (dsPolyh). Three specimens from each group were dissected three days later, corresponding to the second day of the last nymphal instar (N6D2), and the whole body of these individuals (excluding the head and gut), were used to construct and sequence the small RNA libraries. Small RNAs were extracted using the miRNeasy Mini kit (Qiagen), and libraries were prepared using the NEBNext ${ }^{\circledR}$ Multiplex Small RNA Library Prep Set for Illumina ${ }^{\circledR}$ from New England Biolabs. Three library replicates from Control and dsAGO2 treated insects were prepared. Sequencing was carried out at the Genomic Core Facility of the Pompeu Fabra University (Barcelona), using the Illumina NextSeq platform (single strand $\times 50$ cycles). In addition, small RNA libraries from [21], were used to analyze siRNA in dsDcr-1 depleted individuals.

\subsection{RNA extraction, cDNA synthesis and quantitative real-time $P C R$ analysis}

Total RNA was extracted from the whole animals, excluding the head and the digestive tube, using the GenElute ${ }^{\mathrm{TM}}$ Mammalian total RNA kit (Sigma) and RNA concentrations were measured using a nano spectrophotometer (Nabi). One $\mu \mathrm{g}$ of total RNA was then retrotranscribed using the Transcriptor First Strand cDNA Synthesis kit (Roche) as previously described [14]. Primers used to quantify AGO2, Dcr-2, and Actin-5C used as a reference gene were described in [9]. All reactions were run in duplicate or triplicate.

Results from RNA-seq analysis were validated by qRT-PCR using as template a cDNA obtained from the retrotranscription step in the library construction. Levels of three siRNAs corresponding to three different positions in the dsRNA sequence, were measured using the sequence of the siRNA as specific forward primer and the sequence of the adaptor ligated to the $3^{\prime}$ ends during the libraries construction as a common reverse primer as previously described [9]. Primers used to quantify siRNAs along the dsPolyh sequence were: siRNA 1: 5' ATCCTTTCCTGGGACCCGGCAA 3', siRNA 2: 5' TGTTAACGACCAAGAAGTGATG 3' and siRNA 3: 5'CCGACTATGTACCTCATGACGT 3'. As a reference gene, we used U6 [9]. All reactions were run in duplicate or triplicate. 


\section{5. siRNA identification}

Low-quality reads and adapters were removed from the libraries using Trim Galore! V0.6.0 (Babraham Bioinformatics; http://www.bioi nformatics.babraham.ac.uk/projects/trim_galore/). Next, RNA fragments longer than $17 \mathrm{nt}$ were selected and mapped with Bowtie2 v2.2.6 [22] to the fragment of Polyh, AGO2 and Dcr-1 sequence corresponding to the dsRNA used in treatments, forcing zero mismatches on the first 17 nt of the read "-L $17-\mathrm{N}$ 0". Samtools v1.9 [23] was used to calculate the read depth per nucleotide of each strand. Reads were normalized as reads per million reads mapped to $B$. germanica genome plus the sequences of the heterologous dsRNAs (dsPolyh). Read length distribution was calculated using all the reads mapped only in Polyh sequence. The GenomicFeatures software [24] was used for computing the total number of reads mapping to each strand of the injected dsRNAs. In addition, we used the R package seqLogo [25] to visualize the nucleotide proportions in $22 \mathrm{nt}$ length reads.

In order to check if the sorting mechanism for the guide and passenger strands depends on thermodynamic differences between nucleotide pair bonds at the 5'ends of the siRNAs duplexes, we selected the $22 \mathrm{nt}$ sequences showing the highest reads numbers and matched each sequence with the sequence that would correspond to it after being processed by Dcr-2 (two nucleotides overhanging at each 3 'end), whether or not present in our libraries. For the calculation of the bond energies corresponding to the $4 \mathrm{nt}$ at each 5' end we used the ViennaRNA Web Services (http://rna.tbi.univie.ac.at/) [26] (see Supplementary data 2).

Small RNA-seq data are publicly available within the BioProject PRJNA689390.

\subsection{Statistics analysis}

Data from developmental and reproductive parameters, and gene expressions are expressed as mean \pm standard error of the mean (S.E. M.), and statistical differences between data from control and treated animals in these experiments were evaluated using Student's $t$-test with the IBM SPSS Statistics 24 package.

\section{Results}

\subsection{AGO2 mRNA depletion did not produce changes in development and reproduction}

The possible functions of AGO2 in development and reproduction were identified through two different RNAi experiments. In the first experiment, we treated freshly emerged last instar female B. germanica nymphs with dsAGO2, or dsPolyh as a negative control, in order to observe possible effects of the reduction of AGO2 levels in the imaginal molt or in the subsequent reproductive cycle. dsAGO2-treated females presented a significant $85 \%$ decrease in their AGO2 mRNA levels 4 days after treatment (Fig. S1A). However, these AGO2-depleted individuals had no apparent morphological anomalies after molting into adults, and the durations of neither the last nymphal instar nor the first gonadotrophic cycle were affected (Fig. S1A).

In a second approach, we treated freshly emerged adult females with dsAGO2 or dsPolyh. This allowed us to observe the entire first reproductive cycle, and to quantify the duration of the first gonadotrophic cycle and the number of offspring. Again, 4 days after the treatment, AGO2 mRNA levels were $86 \%$ lower in the treated females (Fig. S1B), but we found no differences in either the duration of the first gonadotrophic cycle, or in the number of nymphs that hatched (Fig. S1B).

\subsection{Effect of heterologous dsRNA treatment on AGO2 and Dcr2 expression}

Treating N5D4 with a heterologous dsRNA (dsPolyh) produced an immediate and transient increase in the expression of both AGO2 (Fig. 1A) and Dcr-2 (Fig. 1B). The Dcr-2 mRNA increase was always more prominent than that of $A G O 2$ and this rise was already in evidence $4 \mathrm{~h}$ after the treatment (Fig. 1B). AGO2 mRNA levels showed a 50\% increase $8 \mathrm{~h}$ after the treatment (Figs. 1A). $16 \mathrm{~h}$ after the treatment, mRNAs levels of Dcr2 and AGO2 were not different to their respective controls.

To check whether the observed increase in AGO2 expression is a direct response to the dsRNA, or if $A G O 2$ requires Dcr-2 activity to increase its expression, we treated freshly emerged N5 females with either a dsRNA against $D c r-2$ (dsDcr-2), to prevent the activity of Dcr-2, or with a heterologous dsRNA (dspSTBlue, negative control) to preserve Dcr-2 activity. Four days later, individuals from both treatment groups received an injection of dsPolyh or water, and the Dcr-2 and AGO2 mRNA levels were quantified $8 \mathrm{~h}$ later (Fig. 1C, D and E). The Dcr-2 mRNA levels had decreased in dsDcr-2 animals from both groups (Fig. 1D). The injection of dsPolyh increased Dcr-2 mRNA, in both dspSTBlue and dsDcr-2 individuals, although much lower levels in the case of Dcr-2-depleted animals (Fig. 1D). However, Dcr-2 mRNA levels in dsDcr-2 + dsPolyh subjects were lower than in dspSTBlue $+\mathrm{H}_{2} \mathrm{O}$ animals (Fig. 1D).

AGO2 expression was not significantly affected by $D c r-2$ depletion, and the AGO2 mRNA levels were similar in both dspSTBlue $+\mathrm{H}_{2} \mathrm{O}$ and $d s D c r-2+\mathrm{H}_{2} \mathrm{O}$-treated females (Fig. 1E). Furthermore, dsPolyh injection induced an increase in AGO2 mRNA levels in dspSTBlue + dsPolyh (control) and dsDcr-2 + dsPolyh-treated individuals (23.2\% and 36.4\%, respectively); however, the differences compared with their respective controls were not statistically significant (Fig. 1E). The results therefore indicate that the increased $A G O 2$ expression triggered by treatment with a heterologous dsRNA is not dependent on Dcr-2 activity.

\subsection{Polyh siRNAs analysis in control and dsAGO2 libraries}

To determine how AGO2 is implicated in the occurrence of B. germanica siRNAs, we analyzed the siRNAs produced in the two experimental groups: control individuals, injected sequentially with two heterologous dsRNAs (dspSTBlue + dsPolyh); and AGO2-depleted individuals, injected with dsAGO2 + dsPolyh (Fig. 2A). After assessing the AGO2 mRNA depletion in treated individuals (77\% reduction), and having found no changes in the Dcr2 mRNA levels (Fig. S2), the small RNA libraries were prepared, sequenced, and the siRNAs produced from the dsRNA Polyh used in the treatments were analyzed (Table S1).

All the Polyh reads, from the 3 control and 3 AGO2-depleted libraries, were mapped individually against the sequence of dsRNA Polyh used in the second treatment (Fig. 2B and Table S1). The most common size of the reads mapped to the dsPolyh sequence was $22 \mathrm{nt}$ (Fig. 2C), and the AGO2-depleted libraries had a significant increase in $22 \mathrm{nt}$, around 1.7 times that of the controls (Table S1; Fig. 2C).

The $22 \mathrm{nt}$ reads produced by the treatment with the dsPolyh in both the control and treated libraries, were mapped against the dsPolyh sequence (Fig. 2D). These $22 \mathrm{nt}$ reads were not equally distributed along the entire length of the two RNA strands, and instead were enriched in specific areas (Fig. 2D). The analyses performed mapping the reads between 17 and $51 \mathrm{nt}$ gave a similar profile (Fig. S3). To validate the analysis, we repeated the experiment to construct the libraries. After small RNA extraction and retrotranscription, the relative concentrations of Polyh siRNAs were verified through quantitative real-time PCR, using oligonucleotides designed in Polyh dsRNA regions where the siRNA mapping presents high, intermediate, and low numbers of reads. The results of the qPCR confirmed the abundance differences of the different siRNAs mapped to the Polyh sequence, which had already been observed in the analysis of the libraries (Fig. S3).

Although the distribution of 22 nt Polyh reads appears similar in both the control and dsAGO2-treated specimens, the relative proportions of reads in some dsRNA regions differed between the two treatments (see Fig. 2D). For example, the proportion of reads mapping between nucleotides 180 and 250 on the antisense strand (blue) was 

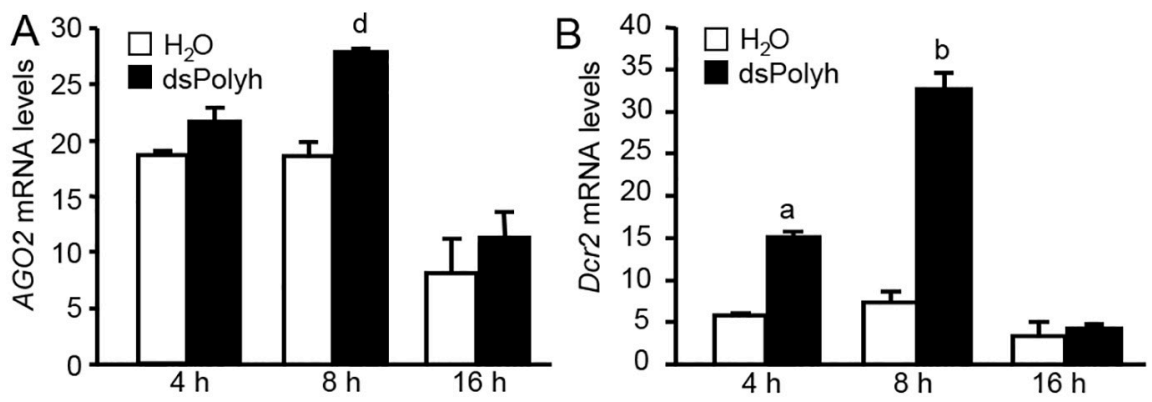

C
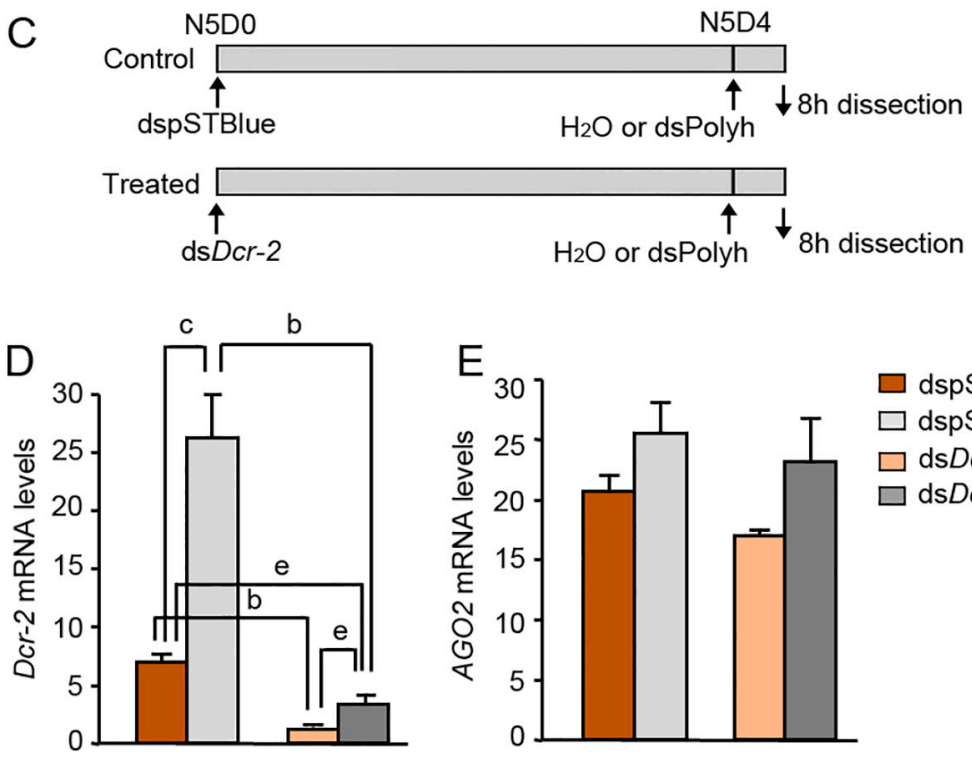

higher in AGO2-depleted individuals than in controls. Additionally, the proportion of reads between nucleotides 80 and 130 on the sense strand (red) was higher in AGO2-depleted individuals (Fig. 2D). These increased proportions point to the abundance of $22 \mathrm{nt}$ reads that are not processed in AGO2-depleted libraries.

To detect any possible differences between the Polyh siRNA sequences from control and AGO2-depleted samples, we analyzed the nucleotide frequency at different positions of the $22 \mathrm{nt}$ siRNA sequences (Fig. 2E and F). In the Polyh siRNAs obtained from the dspSTBlue + dsPolyh (control) treatment, there was a strong preference for a cytosine in the first position at the $5^{\prime}$ end (Fig. 2E). This preference was significantly attenuated in the $A G O 2$ depleted individuals (dsAGO2 + dsPolyh) (Fig. 2F) (frequencies of cytosine in the first position: dspSTBlue + dsPolyh: $0.463 \pm 0.009$; dsAGO2 + dsPolyh: $0.315 \pm 0.005$; Student's $t$ test $p=0.00015$. Results expressed as mean \pm S.E., $n=3$ ).

\section{4. siRNAs from a dsRNAs targeting endogenous B. germanica RNAs}

The previous results were based on an analysis of a heterologous dsRNA. We subsequently wondered if the libraries held examples of siRNAs produced by a dsRNA that targeted endogenous $B$. germanica mRNAs. We therefore analyzed the small RNA libraries, looking for siRNAs corresponding to the dsRNA against AGO2 (dsAGO2) used to deplete AGO2 expression.

In AGO2-depleted libraries, eight days after being treated with dsAGO2, it is still possible to detect a significant number of siRNA reads corresponding to this dsRNA (Table S1). These siRNAs were mapped against a AGO2 dsRNA sequence (Fig. 4A) and, in a similar way to that seen with Polyh siRNAs, the 22 nt reads of $A G O 2$ were not distributed

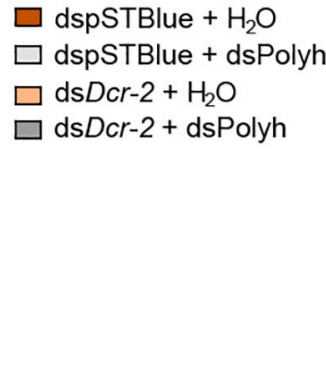

Fig. 1. Effect of the treatment with a heterologous dsRNA on the expression of RNAi pathway enzymes. A-B. 4-day-old penultimate instar nymphs (N5D4) were treated with $2 \mu \mathrm{g}$ of dsPolyh or with the equivalent volume of water. The mRNA levels of AGO2 (A) and Dcr-2 (B) were measured 4, 8 and $16 \mathrm{~h}$ later. C-E. Freshly emerged penultimate instar nymph (N5D0) were treated with $2 \mu \mathrm{g}$ of dsDcr-2 or $2 \mu \mathrm{g}$ of a heterologous dsRNA prepared using the pSTBlue-1 (dspSTBlue). Four days later, individuals of both treatments received an injection of $2 \mu \mathrm{g}$ of dsPolyh or the equivalent volume of water (C). Dcr-2 (D) and AGO2 (E) mRNA levels were measured $8 \mathrm{~h}$ later. Data represent copies of mRNA per 1000 copies of actin- $5 c$ and are expressed as the mean \pm S.E.M. $(n=3-5)$. Statistical differences to the respective control are indicated (Student's $t$-test, a: $p<0.0001$; b: $p<0.0005$; c: $p<0.001$; d: $p<0.005$; e: $p<0.05$ ).

homogenously along the dsAGO2 sequence, but instead enriched certain areas of the dsRNA, with a different pattern to that observed for the siRNAs mapping to the Polyh dsRNA sequence (Fig. 3A). In addition, in the animals treated with dsAGO2, we could only find 22 nt reads covering the area included in the dsRNA used in the treatments and we didn't detect reads mapping in other regions of the AGO2 mRNA or in the gene introns (Fig. S4).

We also analyzed the small RNA libraries produced by Ylla et al. [21], from B. germanica individuals treated with dsRNA against $D c r-1$. In these libraries, we were able to identify a high number of $22 \mathrm{nt}$ siRNAs mapping to the dsRNA Dcr-1 sequence (Table S2). These $22 \mathrm{nt}$ siRNAs covered the entire dsRNA sequence, with a distinct pattern that was different to those observed for Polyh and AGO2 dsRNAs (Fig. 3B). Again, we could find reads covering the area where the dsRNA was designed but we could not detect reads in other regions of the Dcr-1 mRNA or in the gene introns (Fig. S4). The siRNAs corresponding to the dsRNA of Polyh, used as dsMock in this experiment, showed a similar pattern to that observed in the experiments presented here (results not shown).

\subsection{Sorting mechanism for the guide vs the passenger strand}

From the siRNA duplexes resulting from the action of Dcr-2, only one strand (the guide strand) is preserved by RISC to silence the complementary RNA $[27,28]$. Using the $22 \mathrm{nt}$ siRNAs identified in our libraries, we tested the reported model, based on D. melanogaster, through which the siRNA sequence retained by the RISC, the guide strand, is the one showing a thermodynamically less stable $5^{\prime}$ end in the siRNA duplex $[7,29]$. To check this model in our samples, we selected the 10-15 siRNAs with the highest number of reads for each treatment. Then, we 
A
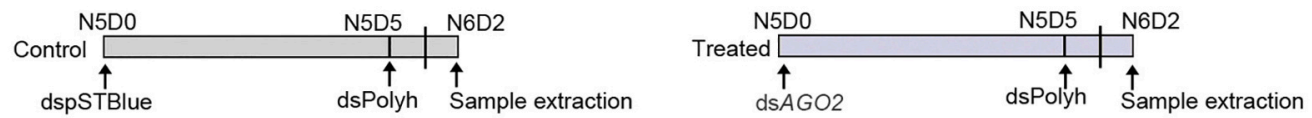

B

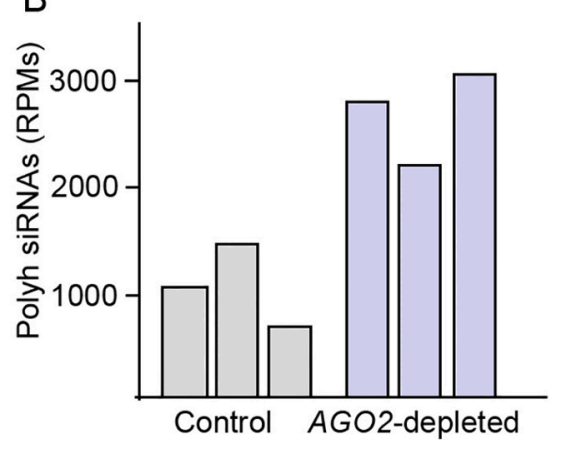

1000
750
500
250

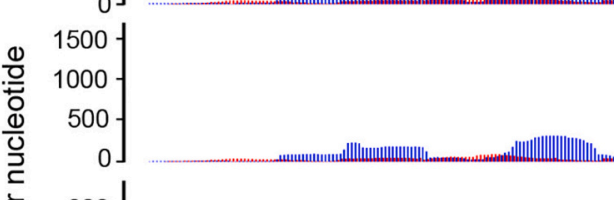

C
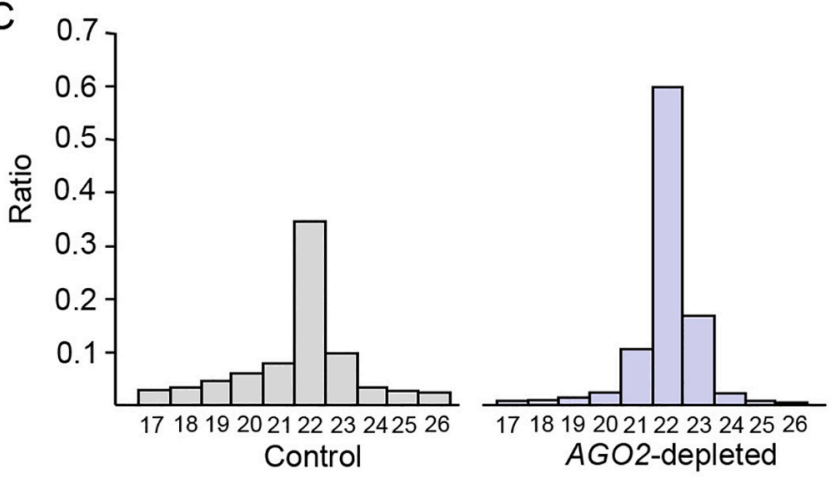

Antisense strand
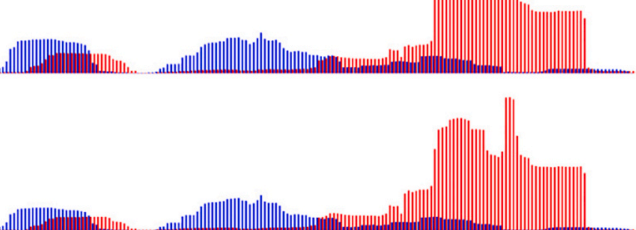

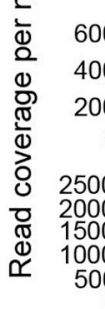

\section{.}
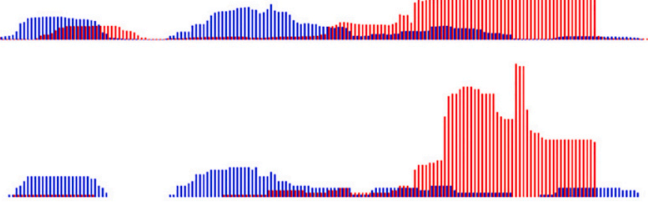

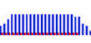

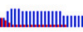
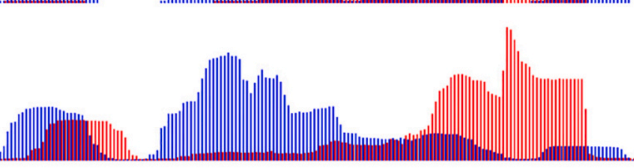

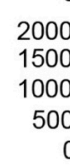

$4000-1$
3000
2000
$1000-1$
0
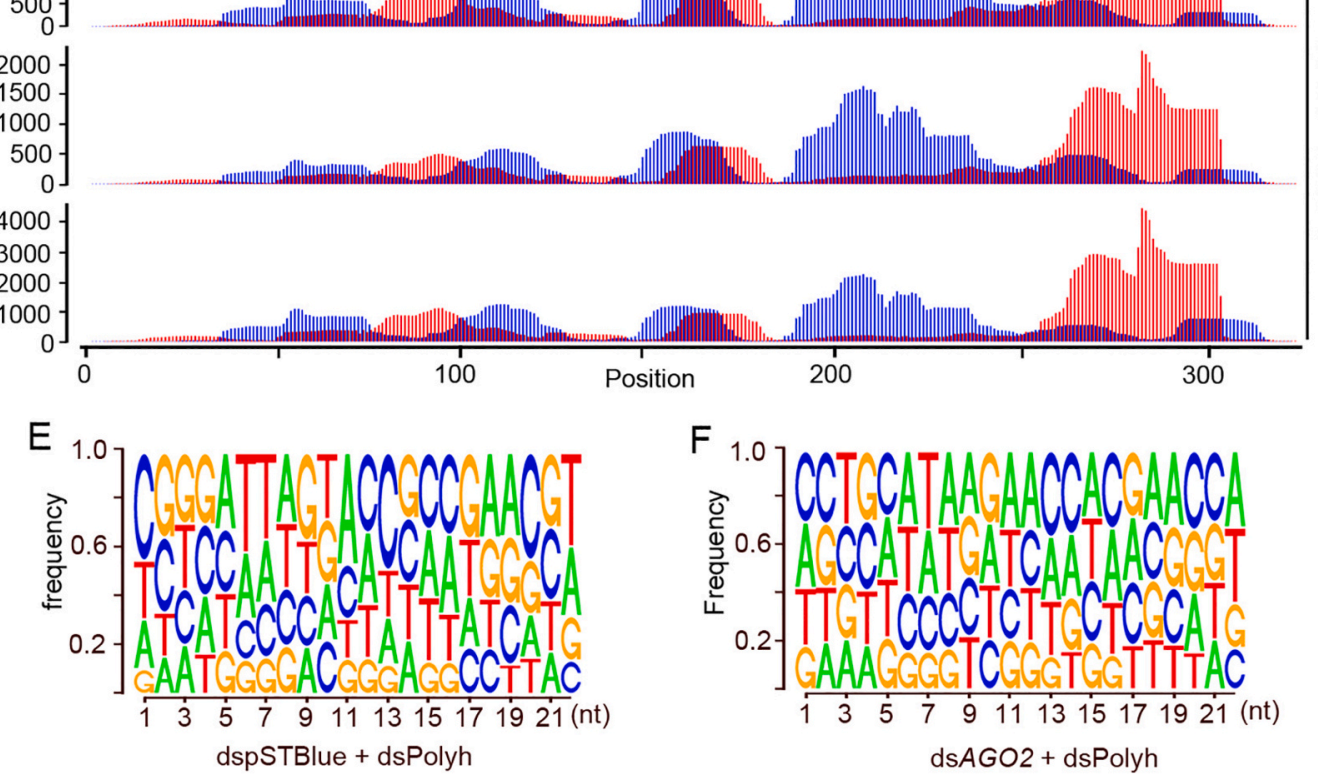

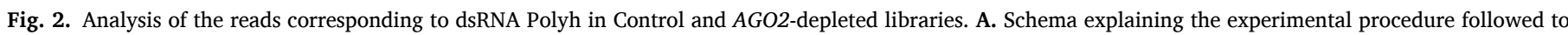

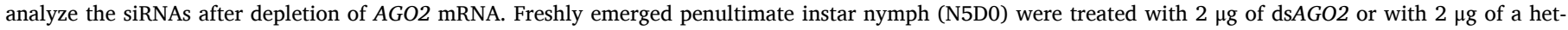

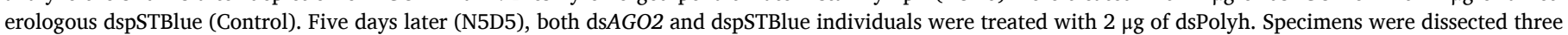

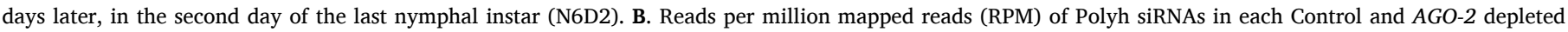

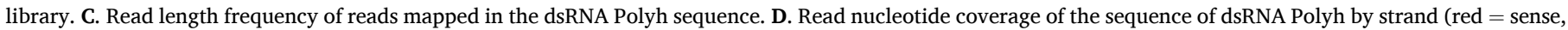

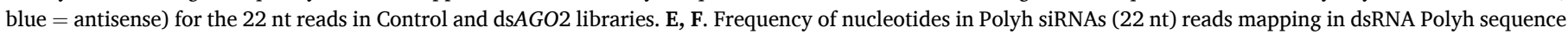

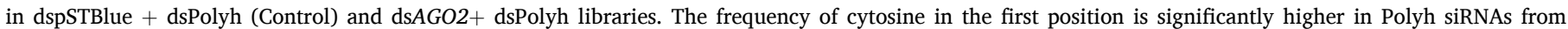
dspSTBlue + dsPolyh than in dsAGO2 + dsPolyh libraries (Student's $t$-test, $p=0.00015$ ). 

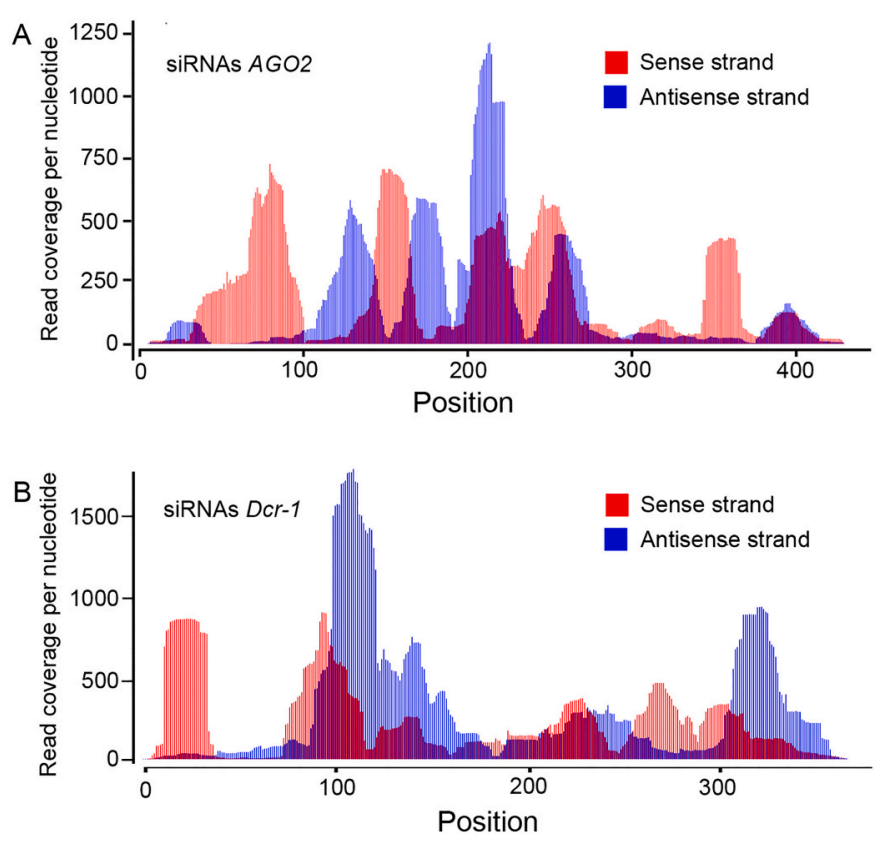

Fig. 3. Analysis of the reads corresponding to dsRNA AGO2 and dsRNA Dcr-1. A. Freshly emerged penultimate instar nymph (N5D0) were treated with $2 \mu \mathrm{g}$ of $\mathrm{ds} A G O 2$ or with $2 \mu \mathrm{g}$ of a heterologous dspSTBlue (Control). Five days later (N5D5), both dsAGO2 and dspSTBlue individuals were treated with $2 \mu \mathrm{g}$ of dsPolyh. Specimens were dissected three days later, in the second day of the last nymphal instar (N6D2). Coverage of the 22 nt reads per nucleotide by strand (red $=$ sense, blue $=$ antisense) of the dsRNA AGO2, from 3 biological replicates together. B. Read coverage per nucleotide by strand (red = sense, blue $=$ antisense) of the dsRNA $D c r-1$ for the $22 \mathrm{nt}$ reads, from 2 biological replicates. These data were obtained from the libraries used in [21], using an equivalent experimental procedure.

identified the complementary sequence that would correspond to its partner in the siRNA duplex, that is, the one that results with two nucleotides overhanging at each $3^{\prime}$ end, whether this sequence was in the library or not (Supplementary data 2). We calculated the bond energy of the four nucleotide pairs at each end, and compared the total energy at one end with that of the other. By checking the number of reads from each strand and the energies at each end, we determined whether the guide and passenger strand selection of the siRNA duplexes corresponding to the most abundant sequences followed the model described for $D$. melanogaster. In $62 \%$ of the duplexes analyzed $(n=34)$ the $\Delta$ energy between the $5^{\prime}$ and $3^{\prime}$ ends of the most abundant sequence of the pair is $>+0.5$, a value compatible with the expected strand selection based on the calculated energies (Supplementary data 2). These results, do not allow us to assert that $B$. germanica follows up the model described for D. melanogaster.

\section{Discussion}

Treating the last instar B. germanica females with a dsRNA against AGO2 did not affect the duration of either the instar or the gonadotrophic cycle. Similarly, depleting AGO2 in adult females did not modify the duration of the gonadotrophic cycle or the number of offspring per female. The results indicate that AGO2 does not play a vital role in either B. germanica metamorphosis or female reproduction, but instead support the idea that its function involves defense against aggression and, if this is absent, the animal's life cycle can proceed normally even when AGO2 levels are reduced. The same applies for other insect species, and similar results have been reported for $D$. melanogaster, where AGO2-mutant flies molt into the adult stage with no apparent problems, with a normal appearance and fertility [30]; and for the western corn rootworm (Diabrotica virgifera virgifera), in which AGO2 depletion has little or no effect on larval growth, adult emergence, survival, or fecundity [31].

Treatment with a heterologous dsRNA (dsPolyh) produced an immediate and transient increase of $A G O 2$ and Dcr-2 expression, which was higher in the case of Dcr-2. This result is similar to that obtained in Manduca sexta larvae treated with a dsRNA, which also produced an increase in Dcr-2 and AGO2 expression, again being much higher in the case of Dcr-2 [32]. This increase in AGO2 expression is not due to increased Dcr-2 or the occurrence of Dcr-2 products (siRNA duplexes), because $D c r-2$ depletion, in the dsDcr-2 treatments, did not preclude the increased AGO2 mRNA levels triggered by the dsPolyh treatment, since similar AGO2 mRNA levels were reached in control (dspSTBlue) and $\mathrm{ds} D c r-2$ animals. For this reason, we presume that there is a yet unknown mechanism for sensing dsRNAs and stimulating the transcription of RNAi pathway components such as Dcr-2 and AGO2. The increased Dcr-2 and AGO2 mRNA levels triggered by dsRNA help the cells to fight the threat generated by the dsRNA agents. In fact, $B$. mori larvae infected with cytoplasmic polyhedrosis virus, a double-stranded RNA virus, also present increased expression of both Dcr-2 and AGO2 [33].

In order to clarify the involvement of AGO2 in siRNA maintenance, we constructed small RNA libraries from control and dsAGO2 B. germanica-treated individuals and analyzed the siRNAs produced from a dsRNA for Polyh injected, in a second treatment, to both control and treated individuals. The analysis of the reads produced from heterologous dsRNA allowed us to study the activity of the RNAi machinery, regardless of whether the dsRNA had target mRNA or not. The most abundant length for $B$. germanica siRNAs is $22 \mathrm{nt}$, a length included in the range assigned to siRNAs [13], but larger than that seen in $D$. melanogaster or in D. virgifera, where the most abundant size for siRNAs is $21 \mathrm{nt}$ [34-36]. The model these authors propose for determining the siRNA length involves the fact that the phosphate-binding pocket in the fruit fly Dcr-2 binds the 5'monophosphate of the dsRNA. Then, the C-terminal dsRNA-binding domain (CdsRBD) of Dcr-2 binds to the proximal region of the dsRNA substrate, aligning the dsRNA with the RNase III active site in the RNase III domain [35]. Thus, the distance between the phosphate-binding pocket and the RNase III active site should determine the siRNA length corresponding to $22 \mathrm{nt}$ in the case of B. germanica.

Furthermore, we observed a prevalence of cytosine as the first nucleotide in the case of the $22 \mathrm{nt}$ siRNAs, matching the dsRNA Polyh sequence in the control libraries, but which is greatly attenuated in AGO2-depleted libraries. This fact points to a preference for loading siRNA duplexes in the RISC where cytosine is favored as the first nucleotide in the guide strand. Additionally, in D. melanogaster, AGO2loaded sequences often start with cytosine, both in the case of exo-siRNAs and $\mathrm{miR}^{*}$ when processed through AGO2 instead than by the canonical AGO1-mediated microRNA pathway [29,37].

The siRNAs produced in B. germanica after being treated with a dsRNA do not map homogeneously along the corresponding dsRNA sequences, but instead accumulate in specific areas of the two dsRNA strands. The fact that this occurs for heterologous dsRNA, whose siRNAs have no mRNA targets inside the cells, suggests that the dsRNA cleavage and siRNAs selection is independent of the siRNA activity as a guide for RNA degradation. The heterogeneous distribution of the siRNAs along the dsRNA sequence was also observed for the dsRNA corresponding to AGO2 and Dcr-1, which revealed different siRNA patterns which were also different from that of dsRNA Polyh. These patterns differences between sequences and strands are probably related to the dsRNA sequence used in the study, and point to the difficulty of predicting a priori the siRNAs that are produced and maintained.

A trait observed in the siRNAs produced from dsAGO2 and dsDcr-1 is that there were no siRNAs mapping outside the zone corresponding to the synthesized dsRNA sequence, which suggests the absence of transitive RNAi. Transitive RNAi is a process by which, through the action of a RNA-dependent RNA polymerase (RdRP), secondary siRNAs are synthesized at positions outside the area targeted by the initial dsRNA. Within Metazoa, transitive RNAi has only been described in nematodes 
[38]. Pinzón et al. [39] suggest that RdRP genes were present in the ancestors of numerous Metazoa clades but were subsequently lost at a very high frequency. We have not found any significant BLAST hit to the nematode Caenorhabditis elegans RdRP in the B. germanica genome or transcriptomes suggesting the absence of RdRP orthologs in this insect. Likewise, it has not been possible to detect transitive RNAi neither in the fruit fly nor in the western corn rootworm [12,36,40]. The absence of transitive RNAi allows the specific depletion of mRNA isoforms as, in fact, was possible in the case of the in B. germanica Taiman isoforms [41].

In the case of dsRNA Polyh, the profiles of the accumulated reads observed in the control and dsAGO2 animals were similar, but not identical. In the libraries from dsAGO2 animals, more $22 \mathrm{nt}$ reads were detected, and some of these mapped in areas of the sequence where far fewer reads were found in control animals. The higher proportion of $22 \mathrm{nt}$ siRNAs and their increase in certain areas of the dsRNA sequence in dsAGO2 libraries indicate that, also in B. germanica, AGO2 has a ribonuclease activity that is reduced in the case of dsAGO2 animals. In addition, the increase of $22 \mathrm{nt}$ siRNAs and the decrease of larger reads suggest AGO2 acts on Dcr-2 activity regulation.

One important step in RNAi is the selection of the guide strand from the siRNA duplex released after Dcr-2 digestion. In D. melanogaster, R2D2 participates in this process before AGO2 within the RISC takes over the duplex. R2D2 forms a heterodimer with Dcr-2 in such a way that R2D2 binds the siRNA duplex at the extreme with the greatest double-stranded character in terms of bond energy, which defines the orientation of the siRNA duplex when loaded into the RISC [7]. This orientation determines the guide strand as the one presenting the least thermodynamically stable base-pairing at its $5^{\prime}$ end $[7,29]$. In the analysis of our libraries, we found that in $62 \%$ of the selected duplexes with a high energy difference between the nucleotide bonds at the two extremes (see Material and Methods and supplementary data), the strand with the highest number of reads is the one with the least thermodynamically stable base-pairing at its $5^{\prime}$ end, as in the proposed model. However, in the remaining cases, a considerably high proportion, the other strand is the one with the highest number of reads. For this reason, although it is possibly the case, we cannot fully assert that the model proposed for D. melanogaster is fulfilled in B. germanica, and we cannot discard the possibility that in this species, and perhaps in other insects, the guide strand selection mechanism is not the same as in D. melanogaster, a point that should be studied in more detail.

According to our results and those provided in the literature involving studies of other species, particularly $D$. melanogaster, we propose that in B. germanica, when the cells detect a dsRNA, there is a rapid and transient increase in the expression of Dcr-2 and AGO2 triggered by a yet unknown mechanism that senses the dsRNA and activates RNAi machinery. AGO2 expression is activated by the presence of a dsRNA in the cell, and not by Dcr- 2 or the siRNA duplexes produced by Dcr-2 activity, which are the substrate for AGO2 activity. Dcr-2 then cleaves the dsRNA producing $22 \mathrm{nt}$ siRNA duplexes. Dcr-2, in a separate process to dsRNA cleavage [42,43], and together with the double-stranded RNA binding protein R2D2, loads the siRNA duplex into the RISC [7,44].

Although we could not identify the mechanism determining guide strand selection, we did observe a clear preference for some strands over others. We cannot rule out the possibility that guide strands are selected according to thermodynamic criteria [7,29], but we did detect a slight preference for cytosine as the first nucleotide of the guide strand. When depleting AGO2 through RNAi (dsAGO2), more 22 nt siRNAs, corresponding to dsRNA areas that are less well-represented in the case of the controls, were accumulated due to the reduction of the AGO2 RNase activity.

Upcoming studies that increase our knowledge of RNAi machinery and mechanisms will shed even more light on this process that is extremely useful for reverse genetics studies in laboratories, as well as for the design of more effective, specific, and eco-friendly pest-control strategies.
Supplementary data to this article can be found online at https://doi. org/10.1016/j.bbagrm.2021.194704.

\section{Funding}

This work was supported by Spanish Ministry of Economy and Competitiveness and FEDER (grant number CGL2016-76011-R), Secretaria d'Universitats i Recerca, Catalan Goverment (grant number 2017 SGR 1030), Agencia Estatal de Investigación (grant number PID2019104483GB-I00/AEI/10.13039/501100011033), and Agencia Estatal Consejo Superior de Investigaciones Científicas (grant numbers 2019AEP028 and 2019AEP029).

\section{CRediT authorship contribution statement}

JCM: Bioinformatic analysis design and performance.

CR: Physiological analysis.

GY: Bioinformatic analysis design and performance. General conceptualization.

MDP: General conceptualization. Supervision. Writing. Funding acquisition.

JLM: General conceptualization. Supervision. Writing. Funding acquisition.

All authors read and approved the final manuscript.

\section{Declaration of competing interest}

The authors declare that they have no known competing financial interests or personal relationships that could have appeared to influence the work reported in this paper.

\section{References}

[1] B. Czech, G.J. Hannon, Small RNA sorting: matchmaking for Argonautes, Nat. Rev. Genet. 12 (2011) 19-31.

[2] X. Bellés, Beyond Drosophila: RNAi in vivo and functional genomics in insects, Annu. Rev. Entomol. 55 (2010) 111-128.

[3] S. Yan, B. Ren, B. Zeng, J. Shen, Improving RNAi efficiency for pest control in crop species, Biotechniques 68 (2020) 283-290.

[4] M. Zotti, E.A. dos Santos, D. Cagliari, O. Christiaens, C.N.T. Taning, G. Smagghe, RNA interference technology in crop protection against arthropod pests, pathogens and nematodes, Pest Manag. Sci. 74 (2018) 1239-1250.

[5] Y.-H. Lin, J.-H. Huang, Y. Liu, X. Belles, H.-J. Lee, Oral delivery of dsRNA lipoplexes to German cockroach protects dsRNA from degradation and induces RNAi response, Pest Manag. Sci. 73 (2017) 960-966.

[6] J. Lozano, E. Gomez-Orte, H.-J. Lee, X. Belles, Super-induction of Dicer-2 expression by alien double-stranded RNAs: an evolutionary ancient response to viral infection? Dev. Genes Evol. 222 (2012) 229-235.

[7] Y. Tomari, C. Matranga, B. Haley, N. Martinez, P.D. Zamore, A protein sensor for siRNA asymmetry, Science 306 (2004) 1377-1380.

[8] X. Zhou, Z. Liao, Q. Jia, L. Cheng, F. Li, Identification and characterization of Piwi subfamily in insects, Biochem. Biophys. Res. Commun. 362 (2007) 126-131.

[9] M. Rubio, J.L. Maestro, M.-D. Piulachs, X. Belles, Conserved association of Argonaute 1 and 2 proteins with miRNA and siRNA pathways throughout insect evolution, from cockroaches to flies, Biochim. Biophys. Acta - Gene Regul. Mech. 1861 (2018) 554-560.

[10] Y. Kawamura, K. Saito, T. Kin, Y. Ono, K. Asai, T. Sunohara, T.N. Okada, M. C. Siomi, H. Siomi, Drosophila endogenous small RNAs bind to Argonaute 2 in somatic cells, Nature 453 (2008) 793-797.

[11] T.A. Rand, K. Ginalski, N.V. Grishin, X. Wang, Biochemical identification of Argonaute 2 as the protein required for RNA-induced silencing complex activity, Proc. Natl. Acad. Sci. U. S. A. 101 (2004) 14385-14389.

[12] S.M. Elbashir, W. Lendeckel, T. Tuschl, RNA interference is mediated by 21- and 22-nucleotide RNAs, Genes Dev. 15 (2001) 188-200.

[13] S.M. Elbashir, J. Martinez, A. Patkaniowska, W. Lendeckel, T. Tuschl, Functional anatomy of siRNAs for mediating efficient RNAi in Drosophila melanogaster embryo lysate, EMBO J. 20 (2001) 6877-6888.

[14] J. Castro-Arnau, A. Marín, M. Castells, I. Ferrer, J.L. Maestro, The expression of cockroach insulin-like peptides is differentially regulated by physiological conditions and affected by compensatory regulation, J. Insect Physiol. 114 (2019) 57-67.

[15] P. Irles, N. Elshaer, M.D. Piulachs, The Notch pathway regulates both the proliferation and differentiation of follicular cells in the panoistic ovary of Blattella germanica, Open Biol. 6 (2016). 
[16] M. Abrisqueta, S. Süren-Castillo, J.L. Maestro, Insulin receptor-mediated nutritional signalling regulates juvenile hormone biosynthesis and vitellogenin production in the German cockroach, Insect Biochem. Mol. Biol. 49 (2014) 14-23.

[17] P. Irles, S. Ramos, M.-D. Piulachs, SPARC preserves follicular epithelium integrity in insect ovaries, Dev. Biol. 422 (2017) 105-114.

[18] S. Ramos, F. Chelemen, V. Pagone, N. Elshaer, P. Irles, M.D. Piulachs, Eyes absent in the cockroach panoistic ovaries regulates proliferation and differentiation through ecdysone signalling, Insect Biochem. Mol. Biol. 123 (2020).

[19] C.V. Dominguez, J.L. Maestro, Expression of juvenile hormone acid O -methyltransferase and juvenile hormone synthesis in Blattella germanica, Insect Sci. 25 (2018) 787-796.

[20] J.L. Maestro, J. Cobo, X. Bellés, Target of rapamycin (TOR) mediates the transduction of nutritional signals into juvenile hormone production, J. Biol. Chem. 284 (2009) 5506-5513.

[21] G. Ylla, B. Fromm, M.-D. Piulachs, X. Belles, D.P. Bartel, E. Berezikov, N. Bushati, S. Cohen, H. Herranz, S.M. Cohen, K.J. Peterson, M.R. Dietrich, M.A. McPeek, J، E. Tarver, X. Belles, K.L. Lucas, B. Zhao, L. Liu, A.S. Raikhel, J. Lozano, et al., The microRNA toolkit of insects, Sci, Rep. 6 (2016) 37736.

[22] B. Langmead, S.L. Salzberg, Fast gapped-read alignment with Bowtie 2, Nat. Methods 9 (2012) 357-359.

[23] H. Li, B. Handsaker, A. Wysoker, T. Fennell, J. Ruan, N. Homer, G. Marth, G. Abecasis, R. Durbin, The sequence alignment/map format and SAMtools, Bioinformatics 25 (2009) 2078-2079.

[24] M. Lawrence, W. Huber, H. Pagès, P. Aboyoun, M. Carlson, R. Gentleman, M. T. Morgan, V.J. Carey, Software for computing and annotating genomic ranges, PLoS Comput. Biol. 9 (2013), e1003118.

[25] O. Bembom, Sequence Logos for DNA Sequence Aligments, 2019.

[26] P. Kerpedjiev, S. Hammer, I.L. Hofacker, Forna (force-directed RNA): simple and effective online RNA secondary structure diagrams, Bioinformatics 31 (2015) 3377-3379.

[27] K. Kim, Y.S. Lee, D. Harris, K. Nakahara, R.W. Carthew, The RNAi Pathway Initiated by Dicer-2 in Drosophila, Cold Spring Harb. Symp. Quant. Biol., Cold Spring Harb Symp Quant Biol, 2006, pp. 39-44.

[28] A. Dueck, G. Meister, Assembly and function of small RNA-Argonaute protein complexes, biol, Chem. 395 (2014) 611-629.

[29] B. Czech, R. Zhou, Y. Erlich, J. Brennecke, R. Binari, C. Villalta, A. Gordon, N. Perrimon, G.J. Hannon, Hierarchical rules for Argonaute loading in Drosophila, Mol. Cell 36 (2009) 445-456.

[30] K. Okamura, A. Ishizuka, H. Siomi, M.C. Siomi, Distinct roles for Argonaute proteins in small RNA-directed RNA cleavage pathways, Genes Dev. 18 (2004) 1655-1666.
[31] C. Davis-Vogel, A. Ortiz, L. Procyk, J. Robeson, A. Kassa, Y. Wang, E. Huang, C. Walker, A. Sethi, M.E. Nelson, D.G. Sashital, Knockdown of RNA interference pathway genes impacts the fitness of western corn rootworm, Sci. Rep. 8 (2018).

[32] J.S. Garbutt, S.E. Reynolds, Induction of RNA interference genes by doublestranded RNA; implications for susceptibility to RNA interference, Insect Biochem. Mol. Biol. 42 (2012) 621-628.

[33] A. Kolliopoulou, F. Van Nieuwerburgh, D.J. Stravopodis, D. Deforce, L. Swevers, G. Smagghe, Transcriptome analysis of Bombyx mori larval midgut during persistent and pathogenic cytoplasmic polyhedrosis virus infection, PLoS One 10 (2015), e0121447.

[34] S.K. Kandasamy, R. Fukunaga, Phosphate-binding pocket in Dicer-2 PAZ domain for high-fidelity siRNA production, Proc. Natl. Acad. Sci. U. S. A. 113 (2016) 14031-14036.

[35] S.K. Kandasamy, L. Zhu, R. Fukunaga, The C-terminal dsRNA-binding domain of Drosophila Dicer-2 is crucial for efficient and high-fidelity production of siRNA and loading of siRNA to Argonaute2, RNA 23 (2017) 1139-1153.

[36] H. Li, A.J. Bowling, P. Gandra, M. Rangasamy, H.E. Pence, R.E. McEwan, C. Khajuria, B.D. Siegfried, K.E. Narva, Systemic RNAi in western corn rootworm, Diabrotica virgifera virgifera, does not involve transitive pathways, Insect Sci. 25 (2018) 45-56.

[37] M. Ghildiyal, J. Xu, H. Seitz, Z. Weng, P.D. Zamore, Sorting of Drosophila small silencing RNAs partitions microRNA* strands into the RNA interference pathway, RNA 16 (2010) 43-56.

[38] T. Sijen, J. Fleenor, F. Simmer, K.L. Thijssen, S. Parrish, L. Timmons, R.H. A. Plasterk, A. Fire, On the role of RNA amplification in dsRNA-triggered gene silencing, Cell 107 (2001) 465-476.

[39] N. Pinzón, S. Bertrand, L. Subirana, I. Busseau, H. Escrivá, H. Seitz, Functional lability of RNA-dependent RNA polymerases in animals, PLoS Genet. 15 (2019), e1007915.

[40] J.Y. Roignant, C. Carré, B. Mugat, D. Szymczak, J.A. Lepesant, C. Antoniewski, Absence of transitive and systemic pathways allows cell-specific and isoformspecific RNAi in Drosophila, RNA 9 (2003) 299-308.

[41] J. Lozano, T. Kayukawa, T. Shinoda, X. Belles, A role for Taiman in insect metamorphosis, PLoS Genet. 10 (2014), e1004769.

[42] K. Förstemann, M.D. Horwich, L. Wee, Y. Tomari, P.D. Zamore, Drosophila microRNAs are sorted into functionally distinct Argonaute complexes after production by Dicer-1, Cell 130 (2007) 287-297.

[43] Y. Tomari, T. Du, P.D. Zamore, Sorting of Drosophila small silencing RNAs, Cell 130 (2007) 299-308.

[44] K. Okamura, N. Robine, Y. Liu, Q. Liu, E.C. Lai, R2D2 organizes small regulatory RNA pathways in Drosophila, Mol, Cell. Biol. 31 (2011) 884-896. 